\title{
Access Strategies for Spectrum Sharing in Fading Environment: Overlay, Underlay, and Mixed
}

\author{
Mohammad G. Khoshkholgh, Student Member, IEEE, \\ Keivan Navaie, Senior Member, IEEE, and Halim Yanikomeroglu, Member, IEEE
}

\begin{abstract}
In this paper, we analyze the achievable capacity of the secondary service for overlay and underlay access strategies. We then propose a novel mixed access strategy in which in contrast to the underlay strategy, the secondary service transmits during the idle periods without considering the interference threshold constraint. In contrast to the overlay strategy, mixed strategy makes transmission during the busy periods with a probability $p_{a}$ subject to satisfying the interference threshold constraint. Parameter $p_{a}$ is a secondary service parameter, which can be adjusted based on the spectrum status. Moreover, we show that the secondary service can adjust $p_{a}$ to select appropriate access strategy with the objective of maximizing the achieved capacity based on the interference at the secondary service receiver, $I$, imposed by the primary service transmitter. The proposed spectrum-sharing technique developed in this paper based on $I$ significantly reduces the system complexity comparing to the system in which for spectrum sharing, the imposed interference at the primary receiver is required. We further suggest a simple power allocation scheme for the mixed strategy that its achieved capacity is very close to the maximum achievable capacity of the secondary service.
\end{abstract}

Index Terms-Achievable capacity, dynamic spectrum access, interference threshold, overlay strategy, spectrum sharing, underlay strategy.

\section{INTRODUCTION}

T spectrum sharing, under certain conditions, a Secondary 1 Service is able to access to a frequency band formally allocated to the Primary Service [1], [2]. Various schemes are proposed in the literature for spectrum sharing (see, e.g., [3]), where the secondary service dynamically detects and makes use of the spectrum holes or white spaces [2], [4], and [5]. White spaces are those parts of the spectrum allocated to the primary user which are under-utilized in some particular times and specific locations. In most cases, spectrum sharing is required to be implemented without imposing changes to the primary network and/or intersystem signaling [2], [3].

The interference threshold or interference temperature constraint is introduced by the Federal Communications Commission (FCC) [6] which indicates the tolerable interference level at the primary receiver imposed by the operation of the secondary service. Technically, as long as the interference threshold constraint is satisfied, the spectrum is underutilized.

- M.G. Khoshkholgh and K. Navaie are with the Wireless Innovation Laboratory (WIL), Department of Electrical and Computer Engineering, Tarbiat Modares University, PO Box 14115-349, Tehran, Iran.

E-mail: \{khoshkholgh, navaie\}@modares.ac.ir.

- H. Yanikomeroglu is with the Broadband Communications and Wireless Systems (BCWS) Centre, Department of Systems and Computer Engineering, Carleton University, Ottawa, ON K1S 5B6, Canada.

E-mail:halim@sce.carleton.ca.

Manuscript received 6 June 2009; revised 8 Nov. 2009; accepted 28 Feb. 2010; published online 17 Mar. 2010.

For information on obtaining reprints of this article, please send e-mail to: tmc@computer.org, and reference IEEECS Log Number TMC-2009-06-0211. Digital Object Identifier no. 10.1109/TMC.2010.57.
In this paper, we focus on the achievable capacity of the secondary service in spectrum sharing. It is shown that obtaining achievable capacity for an Additive White Gaussian Noise (AWGN) channel in different transmission scenarios with interference threshold is an instant of the water-filling problem [7], [8]. The achievable capacity is studied in [9] under various fading conditions. It is shown in [9] that employing opportunistic spectrum sharing results in a significant increase in the achievable capacity in comparison with the spectrum sharing in an equivalent AWGN channel.

In [10], [11], the impact of the interference threshold constraint on the achievable capacity of the secondary service is studied. Moreover, in [12], the secondary system with the multiple-input multiple-output system is considered and the corresponding achievable capacity is obtained. The spectrum access in the spectrum sharing systems which are considered in the aforementioned citations fall in the category of underlay access strategy. ${ }^{1}$ In such systems, the secondary service can always access to the spectrum subject to the interference threshold constraint [3], [13], [14].

Satisfying interference threshold constraint is technically challenging, since the secondary service requires the amount of interference power received at the location of the primary receiver. Moreover, in the underlay strategy, the secondary service must satisfy the interference threshold even in the circumstances in which the primary transmitter is idle. During the idle periods, implying the interference constraint restricts the transmission power of

1. For brevity, we simply refer to "access strategy" as "strategy" in this paper. 
the secondary service, thus reduces its achievable capacity. The achievable capacity of the secondary service is further reduced during the busy periods. That is because of the interference imposed by the primary service activity at the secondary service receiver. To tackle the aforementioned issues, overlay spectrum sharing is proposed (see, e.g, [3]).

In overlay spectrum sharing, the secondary service senses the spectrum and conducts transmission in the circumstances in which the spectrum is idle. If the primary service starts transmission, the secondary service is informed by spectrum sensing mechanism and transmission is immediately stopped [3], [13]. Generally, overlay spectrum sharing strategy requires accurate spectrum sensing mechanisms which might be difficult to implement (see, e.g., [15], [16], [17] and references therein). In [18], [19], the spectrum sensing procedures are proposed and the secondary service throughput is studied; however, the presented analysis in [18] and [19] do not consider the impact of the secondary service power allocation on its throughput. In [18], the authors show that there is an optimal sensing time in which the secondary service throughput is maximized, and at the same time, the level of the quality of service (QoS) metrics in the primary network is also guarantied. In [20], a threshold-based spectrum access scheme is proposed to maximize the fraction of time in which the secondary service accesses the spectrum to satisfy the primary service collision probability constraint. It must be noted that in the overlay spectrum sharing, stopping transmission in busy periods, the secondary service ignores the available sharing opportunity which is due to the interference tolerability of the primary service.

The overlay and underlay strategies are compared in [21], [22]. Capacity analysis versus the primary service outage probability in [21] indicates that in license-free bands, the overlay spectrum sharing strategy employing orthogonal channelization, such as Orthogonal Frequency Division Multiplexing (OFDM), offers higher network capacity. Moreover, results in [21] indicate that in the presence of the primary users, the interference temperature constraint limits network capacity in the overlay strategy more than its underlay capacity counterpart. In [23], the impact of the secondary service on the primary systems in the overlay and underlay strategies is studied using stochastic geometry approach. However, a general approach to choose the best access strategy with the objective of maximizing the secondary service achievable capacity has not been proposed in the related literature. It is also suggested in [23] that by combining the underlay and overlay strategies, the negative impacts on the primary service could be decreased. In [24], sensing-based spectrum sharing is proposed. They consider two different power allocation strategies for the secondary service transmitter based on the spectrum sensing output.

As mentioned above, the overlay strategy ignores the interference tolerability of the primary network. Moreover, underlay strategy also ignores the opportunity lied in monitoring the activity of the primary network in which in the idle periods, if detected properly, the secondary service might be able to transmit without interference threshold constraint. Hence, in both underlay and overlay strategies, system-inherent opportunities, which could be exploited to improve the achievable capacity of the secondary service, are missed. Therefore, it is significantly important to find new strategies where the aforementioned features of the primary service operation are properly exploited.

In this paper, we analyze the achievable capacity of the secondary service in the underlay, overlay, and mixed strategies. We then introduce the imposed interference at the secondary service receiver by the primary service transmitter, $I$, as a criterion for finding the capacity-optimal spectrum sharing strategy.

We also propose a mixed strategy that, in contrast to the underlay strategy, the secondary service transmits during the idle periods without considering the interference threshold constraint. We further introduce a new parameter $p_{a}$ as the access probability during the busy periods. In contrast to the overlay strategy, the proposed mixed strategy makes transmission during the busy periods with a probability $p_{a}$ subject to satisfying the interference threshold constraint. Parameter $p_{a}$ is a secondary service parameter which can be adjusted based on the spectrum status.

Based on $I$ in the mixed strategy, we evaluate the optimal average fraction of time within the busy periods, $p_{a}$, where the secondary service should access the spectrum. Numerical results show that based on the value of $I$, the secondary service can adjust $p_{a}$ to choose its access strategy and maximize the achieved capacity. For a large $I$, the overlay strategy is capacity optimal, i.e., mixed strategy with $p_{a}=0$. For a moderate $I$, the capacity optimal strategy is the mixed strategy with $0<p_{a}<1$. For very low $I$, we show that a mixed strategy with $p_{a}=1$, which is actually a modified version of the underlay strategy, is capacity optimal.

The main contribution of this paper is to propose a framework for analyzing access strategies based on the received interference at the secondary service receiver, $I$, as also for analyzing the achievable capacity of the secondary service. The proposed approach to the spectrum sharing based on $I$ significantly reduces the system complexity comparing to the system in which for spectrum sharing, the imposed interference at the primary receiver is required. In many previous works, the impact of $I$ on the secondary service performance is not considered (see, e.g., [9], [11], [25], and [26]). To the best of our knowledge, in the related literature, the work in this paper is the first one that presents a systematic approach which suggests the best access strategy for a given system setting.

The mixed strategy which is proposed in this paper is similar in spirit to the sensing-based spectrum sharing proposed in [24] except that we further introduce a new parameter $p_{a}$ to find the best access probability to the busy period of the spectrum. In [24], a heuristic algorithm is proposed to find the optimal power allocation. The algorithm we propose in this paper is much simpler than the algorithm which is presented in [24]. Simulation results also indicate that almost the same performance is achieved given that the complexity is much lower. It is also shown that the achieved capacity of the proposed power allocation scheme is very close to the maximum achievable capacity of the secondary service. We also provide closed forms for the achieved capacity as well as allocated power.

A dynamic programming approach to maximize the aggregated average achieved capacity of the secondary 
TABLE 1

List of Parameters

\begin{tabular}{l|l}
\hline Parameter & Description \\
\hline$N_{0}$ & power spectral density of the white noise \\
$B$ & total bandwidth of the primary service \\
$\bar{P}_{s}$ & maximum transmit power of $\mathrm{Tx}_{s}$ \\
$Q$ & interference threshold \\
$I$ & average imposed interference from $\mathrm{Tx}_{p}$ at $\mathrm{Rx}_{s}$ \\
$g_{s s}$ & power gain of the channel between $\mathrm{Tx}_{s}$ and $\mathrm{Rx}_{s}$ \\
$g_{s p}$ & power gain of the channel between $\mathrm{Tx}_{s}$ and $\mathrm{Rx}_{p}$ \\
$g_{p s}$ & power gain of the channel between $\mathrm{Tx}_{p}$ and $\mathrm{Rx}_{s}$ \\
$g_{p p}$ & power gain of the channel between $\mathrm{Tx}_{p}$ and $\mathrm{Rx}_{p}$ \\
$p_{i}$ & to the secondary service \\
$\epsilon$ & false-alarm probability \\
$\delta$ & miss-detection probability \\
$\xi$ & maximum allowable miss-detection probability \\
$\xi_{\epsilon}$ & maximum allowable false-alarm probability \\
$\tau$ & available observation time \\
$T$ & packet duration in the secondary service \\
$p_{a}$ & access probability during the busy periods \\
& in the mixed strategy \\
\hline
\end{tabular}

service users based on the primary service outage probability constraint is also developed in [27]. However, in [27], the interference received from the primary service at the secondary service receivers in the busy periods is not considered. They also consider two power transmission levels corresponding to the idle and busy spectrum states. Their proposed algorithm is only feasible for special cases of idle and busy probabilities.

Note that the main objective of this paper is to evaluate the maximum achievable capacity of the secondary service under different access strategies. The maximum achievable capacity provides a practical bound on the achievable capacity given that the parameters are accurate. In cases where the parameters are not accurate, the achievable capacity would be smaller than the maximum achievable capacity bounds obtained in this paper.

The rest of this paper is organized as follows: In Section 2, the system model is presented; then, in Section 3, the achievable capacity of the secondary service in underlay spectrum sharing is analyzed. In Section 4, we then obtain the achievable capacity of the secondary service when the spectrum sharing is conducted based on the overlay strategy. Consequently, in Section 5, we propose the mixed strategy for spectrum sharing and analyze its achievable capacity. The paper is concluded in Section 6.

\section{SySTEM MOdel}

In this section, we describe the system model used in this paper. For easy reference, the parameters utilized throughout the paper are also presented in Table 1 . We consider a $B \mathrm{~Hz}$ wireless flat fading WGN channel. Two services try to access $B \mathrm{~Hz}$ primary spectrum: Primary Service and Secondary Service. The spectrum has been licensed to the primary service. The secondary service does not have the spectrum license; however, it may acquire access to the spectrum based on Opportunistic Spectrum Access (OSA) strategy. There is no direct signaling channel between the primary and secondary services, and the primary network is not aware of the secondary service users. Subscripts $s$ and $p$ are referred to the secondary service, and the primary

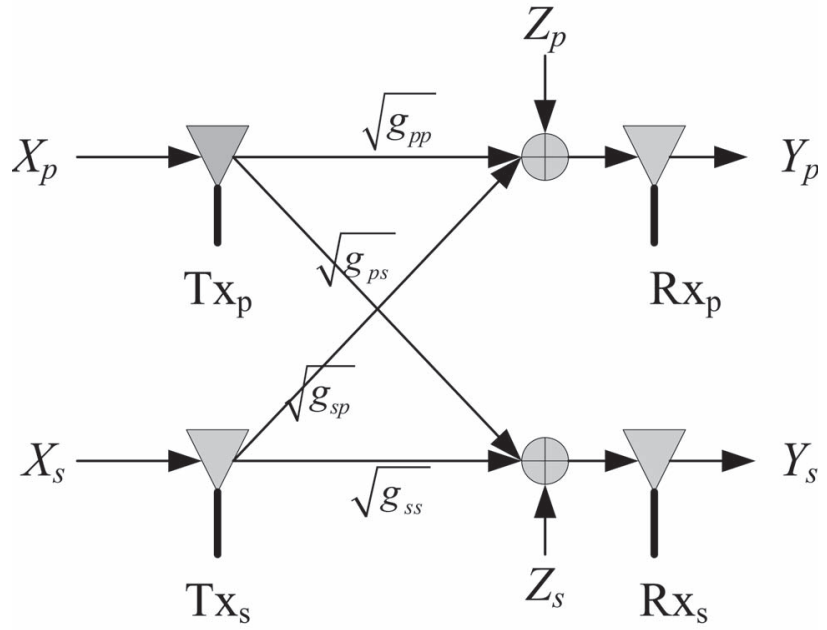

Fig. 1. The schematic of the spectrum sharing system.

service, respectively. Hereafter, we simply refer to "primary spectrum" as "spectrum" unless otherwise stated.

Consider a spectrum sharing scenario with a primary transceiver and a secondary transceiver denoted by $\mathrm{Tx}_{p} / \mathrm{Rx}_{p}$ and $\mathrm{Tx}_{s} / \mathrm{Rx}_{s}$, respectively. Fig. 1 shows the schematic diagram of the spectrum sharing system considered in this paper. Maximum transmit power of $\mathrm{Tx}_{s}$ is assumed to be $\bar{P}_{s}$.

In each time instant $n, g_{s p}[n]$ and $g_{s s}[n]$ denote the instantaneous channel power gains from $\mathrm{Tx}_{s}$ to $\mathrm{Rx}_{p}$, and $\mathrm{Rx}_{s}$, respectively. Similarly, we define $g_{p s}[n]$ and $g_{p p}[n]$ as the instantaneous channel power gains from $\mathrm{Tx}_{p}$ to $\mathrm{Rx}_{s}$, and $\mathrm{Rx}_{p}$, respectively. Therefore, the received signals at $\mathrm{Rx}_{s}$ and $\mathrm{Rx}_{p}$ are represented as

$$
\begin{aligned}
& Y_{s}[n]=\sqrt{g_{s s}[n]} X_{s}[n]+\sqrt{g_{p s}[n]} X_{p}[n]+Z_{s}[n], \\
& Y_{p}[n]=\sqrt{g_{s p}[n]} X_{s}[n]+\sqrt{g_{p p}[n]} X_{p}[n]+Z_{p}[n],
\end{aligned}
$$

where $X_{s}[n]$ and $X_{p}[n]$ are the transmitted signals from $\operatorname{Tx}_{s}$ and $\operatorname{Tx}_{p}$ at time $n$, respectively. In (1) and (2), $Z_{s}[n]$ and $Z_{p}[n]$ are additive white Gaussian noise at $\mathrm{Rx}_{s}$ and $\mathrm{Rx}_{p}$ with variances $\sigma_{s}^{2}$ and $\sigma_{p}^{2}$, respectively. We also assume that $\sigma_{s}^{2}=N_{0} B$. The power channel gains $g_{s s}[n], g_{s p}[n], g_{p p}[n]$, and $g_{p s}[n]$ are assumed to be stationary and ergodic independent random processes with unit-mean probability density (distribution) functions $f_{s s}\left(g_{s s}\right)\left(F_{s s}\left(g_{s s}\right)\right), f_{s p}\left(g_{s p}\right)\left(F_{s p}\left(g_{s p}\right)\right)$, $f_{p p}\left(g_{p p}\right)\left(F_{p p}\left(g_{p p}\right)\right)$, and $f_{p s}\left(g_{p s}\right)\left(F_{p s}\left(g_{p s}\right)\right)$, respectively. For brevity of expositions, hereafter, the time index $n$ is dropped.

Communication activity of the secondary transmitter, $\mathrm{Tx}_{s}$, creates interference at the primary receiver, $\mathrm{Rx}_{p}$, through the channel with power channel gain $g_{s p}$ (see Fig. 1). The maximum tolerable average interference at $\mathrm{Rx}_{p}$ is called interference threshold and is denoted by $Q$. Therefore, the transmitted power at $\mathrm{Tx}_{s}$ should be adjusted so that the interference received at $\mathrm{Rx}_{p}$ is always kept below $Q$. Parameter $Q$ is a system parameter which is usually determined by the band regulator or the primary service operator (see, e.g., [1]).

In this paper, our focus is on the channel small-scale variations. Similar to the previous works, e.g., [9] and [11], the effects of the channel large-scale variations, such as shadowing and distant-dependent path-loss, are scaled into 
the background noise power at the secondary service receiver and the interference threshold constraint.

In the spectrum sharing, the spectrum is either used by the primary services, or remains idle, which are usually called busy and idle periods, respectively. ${ }^{2}$ Generally, the access strategy of the secondary service to the spectrum falls into three following categories: 1) underlay strategy in which spectrum is always accessible to the secondary service subject to an interference constraint at the primary receiver, 2) overlay strategy where the secondary service accesses to the spectrum only during the idle periods, and 3) mixed strategy in which a combination of the aforementioned two strategies is considered. In the following, we investigate the corresponding secondary service achievable capacity for each access strategy.

\section{Underlay StRategy}

As it was mentioned before, in the underlay strategy, the primary service requires the secondary service to always satisfy the interference constraint at $\mathrm{Rx}_{p}$. Therefore, even in circumstances when the primary service is not transmitting (i.e., idle periods), the secondary service has to adjust its transmission power based on the interference threshold constraint. Therefore, in the underlay strategy, the secondary service must always keep the imposed interference at the primary receiver below $Q$; thus, requires to know the channel gains to adjust its transmission power.

Consequently, the maximum achievable capacity of the secondary service in the underlay strategy, $C_{U}$, is the solution of the following optimization problem:

Problem $\mathcal{O}_{U}$.

$$
\begin{aligned}
C_{U}= & \max _{P_{s} \geq 0} \mathbf{E}_{\mathbf{g}} B \log \left(1+\frac{g_{s s} P_{s}}{N_{0} B+g_{p s} P_{p}}\right), \\
\text { s.t. } \quad & \mathbf{E}_{\mathbf{g}} P_{s} \leq \bar{P}_{s}, \\
& \mathbf{E}_{\mathbf{g}} g_{s p} P_{s} \leq Q,
\end{aligned}
$$

where

$$
\mathbf{g} \triangleq(\underbrace{g_{s s}, g_{s p}}_{\mathbf{g}_{s}}, \underbrace{g_{p s}, g_{p p}}_{\mathbf{g}_{p}}),
$$

and $\mathbf{E}_{x}$ represents the expectation with respect to the random variable $x$. In $\mathcal{O}_{U}, P_{s}$ is the transmission power allocated to $\mathrm{Tx}_{s}$ which is generally a function of $\mathrm{g}$. Similarly, $P_{p}$ is the transmission power of $\mathrm{Tx}_{p}$. In the spectrum sharing, the primary service adjusts its transmission power only based on its own channel power gain $g_{p p}$; therefore, $P_{p}$ is only a function of $g_{p p}$.

In $\mathcal{O}_{U}$, the objective function, (3), is Shannon's channel capacity. Moreover, (4) and (5) represent maximum transmission power constraint of $\mathrm{Tx}_{s}$, and interference threshold constraint of $\mathrm{Rx}_{p}$, respectively.

Note that different constraints on the secondary service transmission power and primary service received interference are usually considered in the related literature, for

2. In this paper, when the primary service is inactive (active) the spectrum is called idle (busy). obtaining the secondary service capacity. One may consider constraints on the average or peak transmission power of the secondary service. Similarly, one can also consider constraint of the peak or average received interference at the primary service receiver. For instance, in [11], and [26], various combinations of the average and peak transmission power and received interference are considered, and the corresponding ergodic, delay-limited, and outage capacities are obtained. However, the analysis in [11] and [26] focuses on the underlay strategy. Furthermore, it is shown in [14] and [26] that considering average constraints for secondary service transmit power and primary service received interference results in a higher achieved capacity for the secondary service, while it implies a lower interference on the primary system.

In this paper, we consider the average secondary service transmission power and average primary service received interference, with the objective of finding the access strategy with the highest achievable capacity, as well as analytical tractability. Note that our analysis suggests an upper bound on the secondary service ergodic capacity for different access strategies.

As it was mentioned in Section 2, the channel power gains are independent. We also note that $h(x)=\log \left(1+\frac{a}{b+x}\right)$ is a convex function for $a \geq 0, b \geq 0$, and $x \geq 0$. Therefore, employing Jensen's inequality [28] on the objective function in $\mathcal{O}_{U}$, it can be seen that

$$
\begin{aligned}
\mathbf{E}_{\mathbf{g}} B \log \left(1+\frac{g_{s s} P_{s}}{N_{0} B+g_{p s} P_{p}}\right) \geq \\
\mathbf{E}_{\mathbf{g}_{s}} B \log \left(1+\frac{g_{s s} P_{s}}{N_{0} B+\mathbf{E}_{\mathbf{g}_{p}} g_{p s} P_{p}}\right) .
\end{aligned}
$$

By defining

$$
\begin{aligned}
C_{U}(I) & \triangleq \max _{P_{s} \geq 0} \mathbf{E}_{\mathbf{g}_{s}} B \log \left(1+\frac{g_{s s} P_{s}}{N_{0} B+I}\right), \\
I & \triangleq \mathbf{E}_{\mathbf{g}_{p}} g_{p s} P_{p},
\end{aligned}
$$

and (6), we get an indication that $C_{U} \geq C_{U}(I)$. Therefore, $\mathcal{O}_{U}$ can be approximated as follows:

Problem $\hat{\mathcal{O}}_{U}$.

$$
\begin{aligned}
C_{U}(I)= & \max _{P_{s} \geq 0} \mathbf{E}_{\mathbf{g}_{s}} B \log \left(1+\frac{g_{s s} P_{s}}{N_{0} B+I}\right), \\
\text { s.t. } & \mathbf{E}_{\mathbf{g}_{s}} P_{s} \leq \bar{P}_{s} \\
& \mathbf{E}_{\mathbf{g}_{s}} g_{s p} P_{s} \leq Q
\end{aligned}
$$

where, in this case, $P_{s}$ is a function of $\mathbf{g}_{s}$ and $I$.

Using Lagrange multipliers approach for $\hat{\mathcal{O}}_{U}$, we obtain the optimal transmission power $P_{s}^{*}$ as

$$
P_{s}^{*}=\left(\frac{1}{\lambda_{1}^{*}+\lambda_{2}^{*} g_{s p}}-\frac{N_{0} B+I}{g_{s s}}\right)^{+},
$$

where $(x)^{+}=\max (0, x)$. The Lagrangian coefficients, $\lambda_{1}^{*}$ and $\lambda_{2}^{*}$, are also obtained by replacing $P_{s}^{*}$ into (8) and (9) considering equality. Therefore, 


$$
\int_{\mathcal{G}_{s}}\left(\frac{1}{\lambda_{1}^{*}+\lambda_{2}^{*} g_{s p}}-\frac{N_{0} B+I}{g_{s s}}\right) d F_{s s}\left(g_{s s}\right) d F_{s p}\left(g_{s p}\right)=\bar{P}_{s},
$$

and

$$
\int_{\mathcal{G}_{s}}\left(\frac{g_{s p}}{\lambda_{1}^{*}+\lambda_{2}^{*} g_{s p}}-g_{s p} \frac{N_{0} B+I}{g_{s s}}\right) d F_{s s}\left(g_{s s}\right) d F_{s p}\left(g_{s p}\right)=Q
$$

where

$$
\mathcal{G}_{s} \triangleq\left\{\mathbf{g}_{s}: \frac{g_{s s}}{\lambda_{1}^{*}+\lambda_{2}^{*} g_{s p}} \geq N_{0} B+I\right\}
$$

Using $P_{s}^{*}$ in (10), $C_{U}(I)$ is obtained from (7) as

$$
C_{U}(I)=B \int_{0}^{\infty} \log \left(\frac{x}{N_{0} B+I}\right) d F_{X}(x),
$$

where $F_{X}(x)$ is the PDF of a random variable $x \triangleq \frac{g_{s s}}{\lambda_{1}^{*}+\lambda_{2}^{*} g_{s p}}$. For the case of Rayleigh fading channel, we show in [22] that $f_{X}(x)$ is

$$
f_{X}(x)=e^{-\lambda_{1}^{*} x}\left(\frac{\lambda_{1}^{*}}{1+\lambda_{2}^{*} x}+\frac{\lambda_{2}^{*}}{\left(1+\lambda_{2}^{*} x\right)^{2}}\right) .
$$

\subsection{Power Allocation to the Secondary Service}

As it is seen in (10), $P_{s}^{*}$, among other things, is a function of $g_{s p}$. In other words, to evaluate $P_{s}^{*}, \mathrm{Tx}_{s}$ requires the channel side information (CSI) between itself and $\mathrm{Rx}_{p}$. Providing such information to $\mathrm{Tx}_{s}$ requires either an intersystem signaling, or a third party message-passing scheme which in either cases imposes system overhead. Therefore, in practice, obtaining the optimal power allocation $P_{s}^{*}$ is very complex.

We categorize the underlay spectrum sharing into two following cases: First, the case where $\bar{P}_{s}$ is very high (i.e., there is no practical power constraint for the secondary service), and/or the interference threshold $Q$ is small enough so that for any feasible power allocation holds in (9), the power constraint in (8) is always satisfied. In this case, the dominant constraint is the interference threshold constraint in (9). This assumption is usually made in the literature to find the achievable capacity of the secondary service in underlay strategy (see, e.g., [9] and [11]). In this paper, we refer to this case as Interference Constrained spectrum sharing.

The second case is the one where the interference threshold $Q$ is very high, and/or $\bar{P}_{s}$ is small enough so that the corresponding created interference at the front end of the primary service receiver never crosses the interference threshold, $Q$. In such cases, the interference constraint in (9) is always satisfied. Thus, the dominant constraint is the maximum transmit power constraint of the secondary service in (8). Here, we refer to this case as PowerConstrained spectrum sharing.

\subsubsection{Interference-Constrained Spectrum Sharing}

In interference-constrained spectrum sharing, the constraint (8) in $\widehat{\mathcal{O}}_{U}$ is eliminated. Therefore, $\widehat{\mathcal{O}}_{U}$ is reduced to the following form:

$$
\begin{aligned}
C_{U}^{Q}(I) & =\max _{P_{s} \geq 0} \mathbf{E}_{\mathbf{g}_{s}} B \log \left(1+\frac{g_{s s} P_{s}}{N_{0} B+I}\right), \\
\text { s.t. } & \mathbf{E}_{\mathbf{g}_{s}} g_{s p} P_{s} \leq Q .
\end{aligned}
$$

By utilizing Lagrange Multipliers method, and following the same line of argument as for $\widehat{\mathcal{O}}_{U}$, the optimal transmission power is obtained as

$$
P_{s}^{*}=\left(\frac{1}{\lambda_{Q}^{*} g_{s p}}-\frac{N_{0} B+I}{g_{s s}}\right)^{+},
$$

where $\lambda_{Q}^{*}$ is Lagrangian coefficient and it is obtained by replacing (14) into (13) and performing some straightforward mathematical manipulations.

For Rayleigh fading channel, it is easy to show that $\lambda_{Q}^{*}$ is obtained from the following:

$$
\rho_{Q}-\log \left(1+\rho_{Q}\right)=\gamma_{Q},
$$

where

$$
\begin{aligned}
& \rho_{Q} \triangleq \lambda_{Q}^{*}\left(N_{0} B+I\right), \\
& \gamma_{Q} \triangleq \frac{Q}{N_{0} B+I} .
\end{aligned}
$$

Using (14) and (12), the achievable capacity of the secondary service is

$$
C_{U}^{Q}(I)=B \log \left(1+\rho_{Q}\right) .
$$

Therefore, by increasing $Q, C_{U}^{Q}(I)$ is also increased.

\subsubsection{Power-Constrained Spectrum Sharing}

In the power-constrained spectrum sharing, the interference threshold is always satisfied. Therefore, $\widehat{\mathcal{O}}_{U}$ is converted into the following optimization problem:

$$
\begin{aligned}
C_{U}^{P}(I) & =\max _{P_{s} \geq 0} \mathbf{E}_{\mathbf{g}_{s}} B \log \left(1+\frac{g_{s s} P_{s}}{N_{0} B+I}\right), \\
\text { s.t. } & \mathbf{E}_{\mathbf{g}_{s}} P_{s} \leq \bar{P}_{s} .
\end{aligned}
$$

The above optimization is an instance of water-filling problem. Similarly, utilizing Lagrangian Multiplier method, the optimal transmission power is obtained as follows:

$$
P_{s}^{*}=\left(\frac{1}{\lambda_{P}^{*}}-\frac{N_{0} B+I}{g_{s s}}\right)^{+},
$$

where for the Rayleigh fading channel, $\lambda_{P}^{*}$ is the Lagrangian coefficient which is obtained from

$$
\frac{e^{-\rho_{P}}}{\rho_{P}}-E_{1}\left(\rho_{P}\right)=\gamma_{P},
$$

where

$$
\rho_{P} \triangleq \lambda_{P}^{*}\left(N_{0} B+I\right)
$$

and

$$
\gamma_{P} \triangleq \bar{P}_{s} /\left(N_{0} B+I\right)
$$

In (22), $E_{1}(x)$ is the exponential integral of order 1 defined as $E_{1}(x) \triangleq \int_{1}^{\infty} t^{-1} e^{-x t} d t, \quad x \geq 0$. Using (21) and (19), the achievable capacity of the secondary service is 


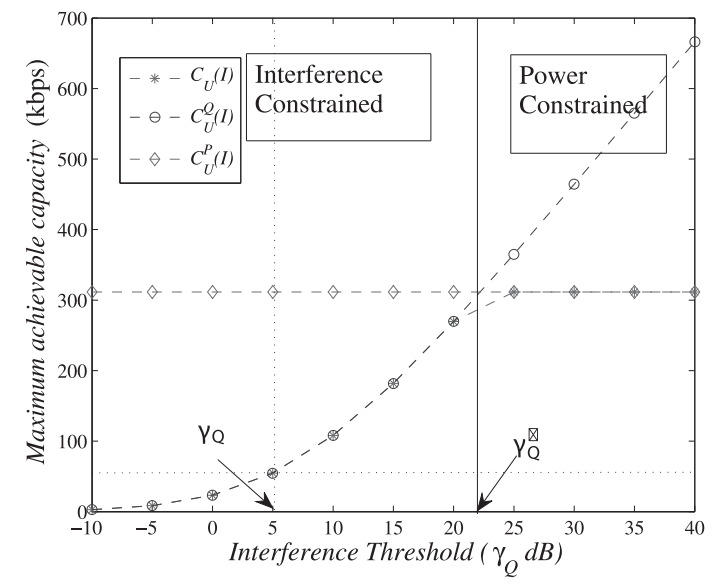

Fig. 2. The achievable capacity of the secondary service for $\bar{P}_{s}=1$ Watt and $I=2 N_{0} B$.

$$
C_{U}^{P}(I)=B \rho_{P} J_{1}\left(\rho_{P}\right),
$$

where $J_{1}(x)$ is defined as $J_{1}(x) \triangleq \int_{1}^{\infty} e^{-x t} \log (t) d t, x \geq 0$. Noting that $E_{1}(x)=x J_{1}(x)$ [29], (23) is simplified as

$$
C_{U}^{P}(I)=B E_{1}\left(\rho_{P}\right) \text {. }
$$

As seen in (24), in power-constrained spectrum sharing, the achievable capacity, $C_{U}^{P}(I)$, is not a function of $Q$.

\subsection{Interference- or Power-Constrained?}

As it is seen, the optimization problem $\widehat{\mathcal{O}}_{U}$, i.e., (7), for interference-constrained, and power-constrained spectrum sharing systems, is reduced into simpler optimization problems (12) and (19), respectively. Thus, identifying the type of the spectrum sharing is critical for system parameters adjustments.

In Fig. 2, $C_{U}(I), C_{U}^{Q}(I)$, and $C_{U}^{P}(I)$ are plotted. As it was mentioned, the achievable capacity $C_{U}^{Q}(I)$ (see (18)) is an increasing function of the interference threshold $Q$ (through $\gamma_{Q}$ ); however, $C_{U}^{P}(I)$ (see (24)) does not change with $\gamma_{Q}$. Therefore, as it is observed in Fig. 2, for a given $\bar{P}_{s}$, or equivalently $\gamma_{P}$, there is a specific amount of $\gamma_{Q}$ where the two capacities $C_{U}^{Q}(I)$ and $C_{U}^{P}(I)$ cross each other. In Fig. 2, this particular point is shown as $\gamma_{Q}^{*}$. Therefore, for the amount of $Q$ where $\gamma_{Q} \leq \gamma_{Q}^{*}$ the spectrum sharing system acts as an interference-constrained spectrum sharing system with the achievable capacity of the secondary service $C_{U}^{Q}(I)$. However, for the case that $\gamma_{Q} \geq \gamma_{Q}^{*}$, the spectrum sharing system acts as a power-constrained spectrum sharing with the achievable capacity $C_{U}^{P}(I)$.

As it is seen in the above discussion, $\gamma_{Q}^{*}$ is a critical system parameter. By obtaining the amount of $\gamma_{Q}^{*}$, we are able to identify whether the spectrum sharing is powerconstrained or interference-constrained. Parameter $\gamma_{Q}^{*}$ can be obtained from (15), (18), (22), and (24), as follows:

$$
\gamma_{Q}^{*}=\gamma_{P}+\left(\rho_{Q}-\frac{e^{-\rho_{P}}}{\rho_{P}}\right) .
$$

According to (25), for a given interference threshold $Q$ (or $\gamma_{Q}$ ) which is the system operating point, it is possible to transform an interference-constrained system to a power-constrained system by adjusting the maximum transmit power, $\bar{P}_{s}$ (or $\left.\gamma_{P}\right)$.

In other words, by adjusting $\bar{P}_{s}$, one can move $\gamma_{Q}^{*}$ to the left-hand side of the actual system operating point, $\gamma_{Q}$, and force the system act as a power-constrained system. Such transformation removes the interference constraint (9), thus, eliminates the need for the channel side information between the secondary transmitter, $\mathrm{Tx}_{s}$, and the primary receiver, $\mathrm{Rx}_{p}$, i.e., $g_{s p}$. Therefore, such transformation significantly reduces the system complexity. It must be noted that by considering an appropriate $\bar{P}_{s}$ so that the new $\gamma_{Q}^{*}$ is smaller than or equal to $\gamma_{Q}$, this transformation does not affect the achievable capacity of the secondary service.

In the following, we consider the overlay strategy for spectrum sharing and analyze its corresponding secondary service achievable capacity.

\section{Overlay Strategy}

In the underlay spectrum sharing strategy, when the primary service is idle, it is still required for the secondary service to satisfy the interference threshold constraint. This constraint limits the transmission power of the secondary service, thus restricts its achievable capacity. This approach has its root in the fact that primary service may start transmission at any arbitrary time instant; therefore, the spectrum should be always available. The spectrum availability is guaranteed through interference threshold satisfaction in both idle and busy periods of the primary service. However, underlay strategy fails to exploit the opportunity lied in monitoring the activity profile of the primary network. In other words, during the idle periods, the secondary service might be able to transmit without interference threshold constraint.

An alterative approach is to ignore the interference threshold during inactive periods of the primary service. However, the main requirement for such approach is to have an online detection of the idle and busy periods with an acceptable level of accuracy. This approach is called overlay spectrum sharing strategy. The procedure of detecting the spectrum status is called spectrum sensing. Note that, in overlay strategy, when the primary service starts transmission, the secondary service must stop transmission immediately.

In the overlay strategy, the amount of imposed interference at $\mathrm{Rx}_{s}$ from $\mathrm{Tx}_{p}$ is reduced to zero, i.e., $I=0$ during the inactive period of the primary service. Note that in the underlay strategy, $I$ is averaged over both inactive and active periods of the primary service; thus, $I \neq 0$. Moreover, eliminating the interference threshold at $\mathrm{Rx}_{p}$ during the inactive periods of the primary service, the only power constraint is the maximum transmission power of the secondary service. Comparing to the underlay strategy, in the overlay strategy, the average time of the spectrum accessability to the secondary service is reduced.

As it was mentioned, in overlay spectrum sharing strategy, the estimation of the spectrum status is very critical. The spectrum sensing mechanisms are not usually perfectly accurate. In this paper, we develop our analysis based on accurate spectrum state estimation in Section 4.1, and then we investigate the impact of inaccurate spectrum state estimation in Section 4.2. 


\subsection{Accurate State Estimation}

We assume that the spectrum is available with the probability $p_{i}$; consequently, the primary service accesses to the spectrum with probability $p_{b}=1-p_{i}$. Therefore, the total secondary service achievable capacity is

$$
C_{O}\left(p_{i}\right)=p_{i} \mathbf{E}_{\mathbf{g}_{s}} B \log \left(1+\frac{g_{s s} P_{s}}{N_{0} B}\right),
$$

and the achievable capacity of the secondary service is obtained from the following optimization problem:

Problem $\mathcal{O}_{O}$.

$$
\begin{aligned}
C_{O}\left(p_{i}\right) & =\max _{P_{s} \geq 0} p_{i} \mathbf{E}_{\mathbf{g}_{s}} B \log \left(1+\frac{g_{s s} P_{s}}{N_{0} B}\right), \\
\text { s.t. } & p_{i} \mathbf{E}_{\mathbf{g}_{s}} P_{s} \leq \bar{P}_{s} .
\end{aligned}
$$

In $\mathcal{O}_{O}$, the average transmission power constraint at $\mathrm{Tx}_{s}$ is modified as in (28) because the secondary service accesses to the spectrum with the probability $p_{i}$.

The problem in $\mathcal{O}_{O}$ is very similar to the optimization problem in (19); however, here $P_{s}$ is only a function of $g_{s s}$. In fact, since in the overlay strategy the secondary service accesses to the spectrum when it is available (i.e., idle), the impact of $g_{s p}$ is eliminated. Moreover, as it is expected, in this case $I=0$. It is worth mentioning that $p_{i}$ actually indicates the average percentage of the total bandwidth $B$ utilized by secondary service. Therefore, by decreasing $p_{i}$ to zero, the achievable capacity $C_{O}\left(p_{i}\right)$ approaches zero as well.

Using Lagrange Multipliers approach, the optimal power allocation of the secondary service with Rayleigh fading is

$$
P_{s}^{*}=\left(\frac{1}{\lambda^{*}}-\frac{N_{0} B}{g_{s s}}\right)^{+},
$$

where the Lagrangian Coefficient $\lambda^{*}$ is obtained from the following equation:

$$
\frac{e^{-\rho}}{\rho}-E_{1}(\rho)=\frac{\gamma_{P}}{p_{i}},
$$

where $\rho \triangleq \lambda^{*} N_{0} B$, and $\gamma_{P} \triangleq \bar{P}_{s} / N_{0} B$.

Consequently, the achievable capacity is

$$
C_{O}\left(p_{i}\right)=p_{i} B E_{1}(\rho),
$$

where $E_{1}(x) \triangleq \int_{1}^{\infty} t^{-1} e^{-x t} d t, \quad x \geq 0$.

In Fig. 3, the achievable capacity of the secondary service in the overlay strategy is plotted versus $p_{i}$. According to this figure, in general, $C_{O}\left(p_{i}\right)$ is increased with increasing $p_{i}$. In this figure, the achievable capacity of the secondary service in the underlay strategy is also plotted for given system parameters, i.e., $I$ and $\gamma_{Q}$. As it is seen, for given $I$ and $\gamma_{Q}$ in the underlay strategy, for $p_{i}<p_{i}^{*}$, the underlay strategy achieves higher capacity and for $p_{i}>p_{i}^{*}$, the overlay strategy achieves higher capacity. The value of $p_{i}^{*}$ where the achievable capacity of the underlay and overlay strategies crosses each other is a function of $I$ and $\gamma_{Q}$. The above discussion suggests that the secondary service should monitor the values of $I, \gamma_{Q}$, and $p_{i}$ and compare the achievable capacity of underlay and overlay strategies, i.e., $C_{U}(I)$ and $C_{O}\left(p_{i}\right)$, and then choose the strategy with the higher achievable capacity.

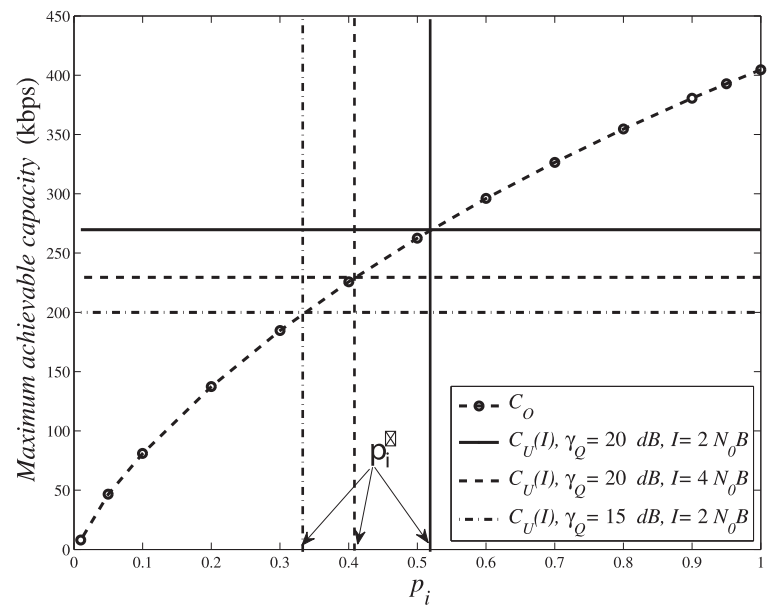

Fig. 3. The achievable capacity of the secondary service in the overlay and underlay spectrum sharing strategies, $\bar{P}_{s}=1 \mathrm{~W}$.

For the case that the spectrum sharing system acts in power constrained region, i.e., $\gamma_{Q} \geq \gamma_{Q}^{*}$, using (24) and (31), $p_{i}^{*}$ is

$$
p_{i}^{*}=\frac{E_{1}\left(\rho_{P}\right)}{E_{1}(\rho)} .
$$

Similarly, for the case that $\gamma_{Q}<\gamma_{Q}^{*}$, i.e., interference-limited spectrum sharing, using (18) and (31) it is seen that

$$
p_{i}^{*}=\frac{\log \left(1+\rho_{Q}\right)}{E_{1}(\rho)} .
$$

Combining the two above equations, we can simply derive $p_{i}^{*}$ as

$$
p_{i}^{*}=\frac{\log \left(1+\rho_{Q}\right)}{E_{1}(\rho)} \mathbf{1}_{\gamma_{Q}<\gamma_{Q}^{*}}+\frac{E_{1}\left(\rho_{P}\right)}{E_{1}(\rho)} \mathbf{1}_{\gamma_{Q} \geq \gamma_{Q}^{*}},
$$

where $\mathbf{1}_{x}=1$, if $x$ is correct, and 0 otherwise. Therefore, if $p_{i}<p_{i}^{*}\left(p_{i} \geq p_{i}^{*}\right)$, the secondary service should switch to the underlay (overlay) strategy.

\subsection{Inaccurate State Estimation}

In practice, spectrum sensing mechanisms are not perfect. Let $\delta$ be the probability of miss-detection, i.e., the probability of estimating the spectrum state as idle, while it is busy, and $\epsilon$ be the probability of false-alarm, i.e., the probability of estimating the spectrum state as busy, while it is idle.

Parameters $\delta$ and $\epsilon$ are specified by the receiver operating characteristic (ROC) curve which gives the probability of detection, $1-\delta$, as a function of $\epsilon$. The ROC is a characteristic of the spectrum sensing technique [3] Using the ROC curve, for a given value of $\delta, \epsilon$ is obtained.

Inaccurate spectrum sharing results in collision between the primary and the secondary service transmissions. Therefore, if the spectrum sharing system is not designed to manage the miss-detection incidents, the collision between the primary and the secondary service transmissions can potentially reduce the actual capacity of the primary network. In practice, however, some sort of tolerability to collision is considered for the primary network; therefore, a collision probability constraint is also considered for the spectrum sharing. In other words, the spectrum sensing mechanism is utilized so that its probability of miss-detection satisfies the collision probability constraint of the primary service, $\xi$. 
Consider the case that the spectrum sensing procedure is based on energy detection. The energy detection scheme is the most popular spectrum sensing procedure [30]. An energy-detector-based spectrum sensing procedure simply measures the energy received due to the primary service activity during an observation interval. The spectrum is considered "idle," if the measured energy is less than a properly set threshold, which is a system parameter.

Let the spectrum sensing procedure be implemented at $\mathrm{Rx}_{s}$. Therefore, the secondary service receiver senses the spectrum based on received interference at $\mathrm{Rx}_{s}$ by the primary service transmission, $I$. On the other hand, when the spectrum sensing procedure is implemented at $\mathrm{Tx}_{s}$, the imposed interference at $\mathrm{Tx}_{s}$ by $\mathrm{Tx}_{p}$ should be also taken into consideration. In the case where the spectrum sensing procedure is implemented at both $\mathrm{Tx}_{s}$ and $\mathrm{Rx}_{s}$, the secondary service accesses to the channel when both of them detect an idle spectrum. In practice, a signaling protocol in the form of request-to-send/clear-to-send (RTS/ CTS) is employed by the secondary service in most cases. Here, we confine our analysis to the spectrum sensing procedure which is only implemented at $\mathrm{Rx}_{s}$.

From the primary service point of view, the best case is $\delta=0$. From the secondary service viewpoint, the ideal spectrum sensing procedure has false-alarm probability equal to zero. However, based on some practical limitations, a tolerance on miss-detection and false-alarm probabilities is acceptable. Let the maximum tolerable collision probability be $\xi$; thus, the secondary service must satisfy $\delta \leq \xi$. In practice, the secondary service limits itself to a false-alarm constraint $\xi_{\epsilon}$, i.e., $\epsilon \leq \xi_{\epsilon}$. Therefore, the energy detector is designed so that the miss-detection and falsealarm constraints are satisfied at the same time.

To derive false-alarm and miss-detection probabilities, usually two hypotheses $\mathcal{H}_{0}$ and $\mathcal{H}_{1}$ are considered, where $\mathcal{H}_{0}$ is the case that no signal from $\operatorname{Tx}_{p}$ is received at $\mathrm{Rx}_{s}$, i.e., only noise is detected, and $\mathcal{H}_{1}$ is the case that in addition to the noise, there are signals from $\mathrm{Tx}_{p}$ received at $\mathrm{Rx}_{s}$. Furthermore, the decision on idle or busy spectrum is made in $\mathrm{Rx}_{s}$ based on comparing the received signal within the observation interval with a decision threshold. The decision threshold is obtained based on $\xi$ and $\xi_{\epsilon}$.

For instance, [18] obtains the miss-detection and falsealarm probabilities for complex-valued PSK as follows:

$$
\begin{aligned}
& 1-\delta=\mathbf{Q}\left(\frac{\mathbf{Q}^{-1}\left(\xi_{\epsilon}\right)-\sqrt{N} \frac{\tau}{T} \frac{p_{b} I}{N_{0} B}}{\sqrt{2 \frac{\tau}{T} \frac{p_{b} I}{N_{0} B}+1}}\right), \\
& \epsilon=\mathbf{Q}\left(\sqrt{2 \frac{\tau}{T} \frac{p_{b} I}{N_{0} B}+1 \mathbf{Q}^{-1}}(1-\xi)+\sqrt{N} \frac{\tau}{T} \frac{p_{b} I}{N_{0} B}\right),
\end{aligned}
$$

where $\mathbf{Q}(x) \triangleq \frac{1}{\sqrt{2 \pi}} \int_{x}^{\infty} e^{\frac{-y^{2}}{2}} d y$, and $N$ is the number of samples in available observation interval, $\tau$, in the secondary service receiver, and $T$ is its packet duration. ${ }^{3}$ For the sampling frequency of $f_{s}$, the number of samples in available sensing time $N$ is $\left\lceil\tau f_{s}\right\rceil$, where $\lceil x\rceil$ is the smallest integer greater than

3. The observation interval $\tau$ is, in fact, the required time in which the sensing mechanism makes decision on the spectrum state. As it is assumed that the spectrum sensing mechanism is implemented in the secondary user, $\tau$ covers any delay corresponding with the spectrum sensing procedure until making a decision on the spectrum status by the secondary service user.

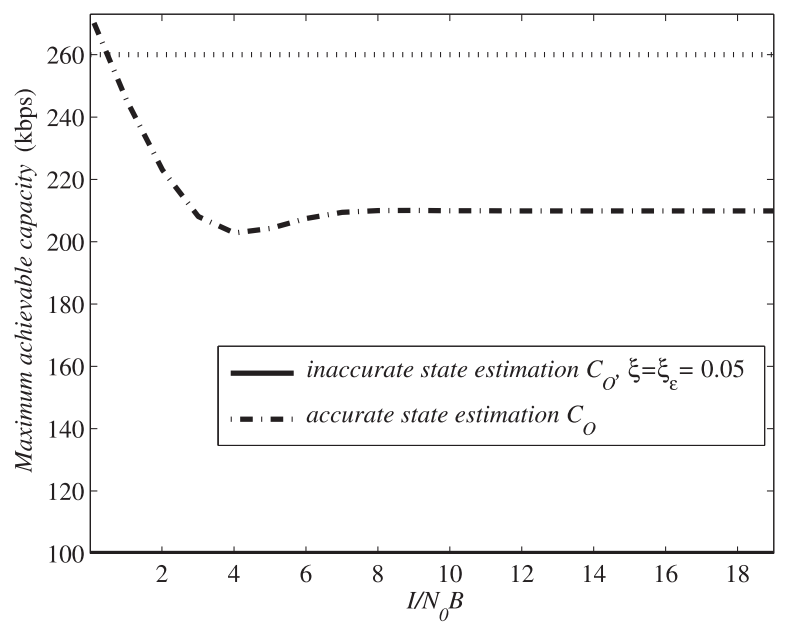

Fig. 4. The achievable capacity of the secondary service for $\bar{P}_{s}=1 \mathrm{~W}$, $p_{i}=0.4$, and $\tau / T=0.1$.

$x$. It is worth mentioning that, in this case, only a fraction $\left(p_{b} \tau / T\right)$ of $I$ is captured during the observation interval in the spectrum sensing procedure.

In case of inaccurate spectrum sensing, during the transmission of each packet, $T$, the secondary service can only transmit within the remaining $T-\tau$ s. Here, we assume that $\tau$ is fixed. One can also consider the case where $\tau$ is assigned adaptively. Therefore, the fraction of time that the secondary service is able to access to channel, $\hat{p}_{i}$, is

$$
\hat{p}_{i}=\left(p_{i}(1-\epsilon)+p_{b} \delta\right) \frac{T-\tau}{T} .
$$

In case of miss-detection, the access time of the secondary service is increased by $p_{b} \delta$. Similarly, in case of false-alarm, the access time of the secondary service decreases by $p_{i}(1-\epsilon)$.

Therefore, the achievable capacity of the secondary service is obtained from the following optimization problem:

Problem $\hat{\mathcal{O}}_{O}$.

$$
\begin{aligned}
C_{O}\left(\hat{p}_{i}\right) & =\max _{P_{s} \geq 0} \hat{p}_{i} \mathbf{E}_{\mathbf{g}_{s}} B \log \left(1+\frac{g_{s s} P_{s}}{N_{0} B+\frac{T-\tau}{T} \delta p_{b} I}\right), \\
\text { s.t. } & \hat{p}_{i} \mathbf{E}_{\mathbf{g}_{s}} P_{s} \leq \bar{P}_{s},
\end{aligned}
$$

where in $\hat{\mathcal{O}}_{O}, \frac{T-\tau}{T} \delta p_{b} I$ is the received interference at the secondary service receiver in the miss-detection case when the spectrum state is wrongly estimated as idle. For brevity, we skip obtaining the solution to this optimization problem, since the solution is similar to that of $\mathcal{O}_{O}$ in the previous section.

\subsection{Numerical Results}

In Fig. $4, C_{O}\left(\hat{p}_{i}\right)$ in (34) is plotted versus $I / N_{0} B$. As it is observed, by increasing $I / N_{0} B$ from 0 to approximately 5 , the achievable capacity is also decreased; however, for $I / N_{0} B>5, C_{O}\left(\hat{p}_{i}\right)$ remains constant. Indeed, by increasing $I / N_{0} B$, on one hand, the accuracy of spectrum sensing procedure is increased, i.e., the false-alarm and missdetection probabilities are decreased, and on the other hand, increasing $I / N_{0} B$ imposes higher interference at the 


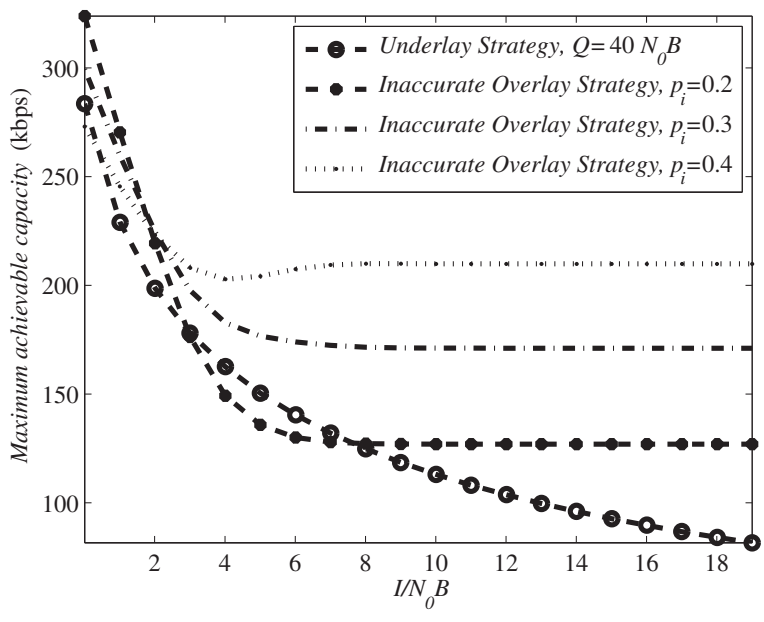

Fig. 5. The achievable capacity of the secondary service in the underlay strategy and overlay strategy with inaccurate spectrum sensing for $\bar{P}_{s}=$ $1 \mathrm{~W}$ and $\xi=0.05$.

secondary service receiver. In cases where $I / N_{0} B<5$, due to less accurate spectrum sensing, increasing interference reduces the achievable capacity; in other words, $C_{O}\left(\hat{p}_{i}\right)$ is decreased by increasing $I / N_{0} B$. However, for $I / N_{0} B>5$, the accuracy of the spectrum sensing procedure is increased, which keeps the achievable capacity constant. This is due to the fact that the product of the miss-detection probability and the imposed interference at the secondary service receiver is almost constant, and this product reduces to zero for this case.

For large values of $I / N_{0} B$, the gap between $C_{O}\left(p_{i}\right)$ and $C_{O}\left(\hat{p}_{i}\right)$ is mainly related to the length of observation interval, $\tau$, and the value of $\epsilon$ which is considered to be $\xi_{\epsilon}$. Consequently, in this case, for large enough $I / N_{0} B$, some available resources for spectrum sharing is not exploited during $\tau$. However, for the case that $I / N_{0} B$ is small, Fig. 4 indicates that $C_{O}\left(\hat{p}_{i}\right)$ is higher than $C_{O}\left(p_{i}\right)$. In this region, high probability of false-alarm and miss-detection make it possible for the secondary service to exploit the busy part of the spectrum and ignore the idle part of it. However, since $p_{b}>p_{i}$ and the value of $I / N_{0} B$ is small in this case, $C_{O}\left(\hat{p}_{i}\right)>C_{O}\left(p_{i}\right)$.

In Fig. 5, we compare the achievable capacity of the secondary service in underlay and overlay access strategies with inaccurate state estimation. As it is seen, by increasing the amount of $I / N_{0} B$, the achievable capacity of the underlay strategy is decreased. The achievable capacity of the overlay strategy is also decreased by increasing $I / N_{0} B$; however, the reduction rate is lower than that of underlay strategy. Based on the system parameters, for lower $I / N_{0} B$, the underlay and overlay strategies result in approximately the same achievable capacity. However, when $I / N_{0} B$ is increased, the robustness of the overlay strategy against $I / N_{0} B$ variations is higher than that of the underlay strategy.

We also compare the underlay and overlay strategies with inaccurate state estimation versus $p_{i}$ in Fig. 6 . This figure suggests that for given system parameters and imposed interference $I$, when $p_{i}$ is small or large enough, the underlay strategy experiences higher achievable capacity. However, for moderate $p_{i}$, the overlay strategy with inaccurate state estimation outperforms the underlay strategy. Therefore, similar to (32), the secondary service should compare its achievable capacity in underlay strategy and

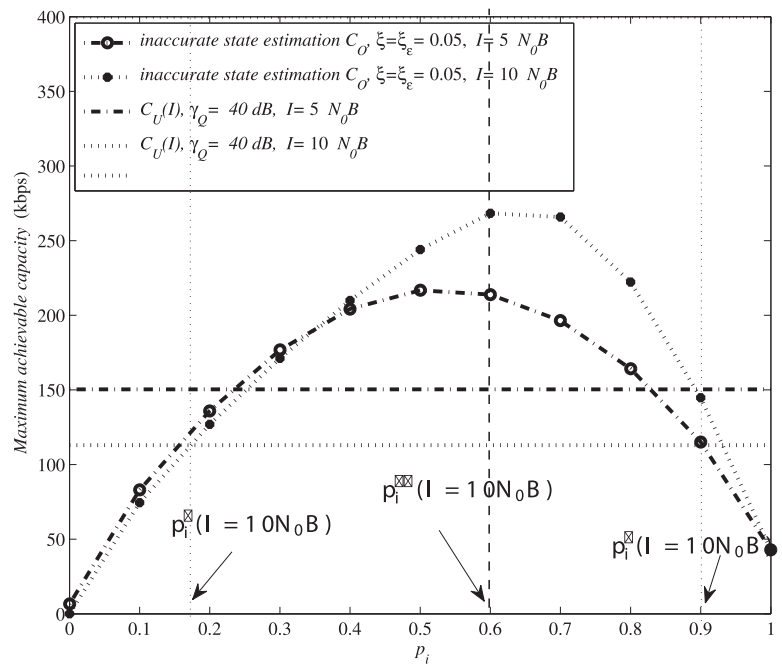

Fig. 6. The achievable capacity of the secondary service in the underlay strategy and overlay strategy with inaccurate spectrum sensing versus $p_{i}$ for $\bar{P}_{s}=1 \mathrm{~W}, I=10 N_{0} B$, and $\xi=0.05$.

overlay strategy with inaccurate spectrum sensing and then find $p_{i}^{*}$. The best access strategy is then chosen based on that.

Fig. 6 also indicates that in cases where the dominate access strategy is overlay with inaccurate spectrum sensing, there is an optimal value of $p_{i}, p_{i}^{* *}$ for given system parameters that maximizes the secondary service capacity. However, it must be noted that, by increasing $I$ from zero to infinity, the value of $p_{i}^{* *}$ decreases from 1 to 0 . Indeed, when $I=0$, the spectrum is considered idle; therefore, $p_{i}^{* *}=1$.

Based on the presented analysis in the two previous sections, it is observed that the overlay and underlay strategies outperform each other in different circumstances. This raises an important question: is it possible for the secondary service to adaptively select the best access strategy? Based on the presented analysis in the previous sections, the secondary service might be able to choose the optimal strategy based on the primary service situation, i.e., $p_{i}, I$, and its interference constraint, $Q$.

In the following section, we elaborate this issue and propose a mixed strategy with the objective of maximizing the achievable capacity of the secondary user.

\section{Mixed Strategy}

In contrast to the underlay strategy, based on the proposed mixed strategy, the secondary service transmits without considering the interference threshold constraint during the idle periods. Moreover, during busy periods, the proposed mixed strategy, in contrast to the overlay strategy, the secondary service makes transmission with a probability $p_{a}$ subject to satisfying the interference threshold constraint. Parameter $p_{a}$ is a secondary service parameter which can be adjusted based on the spectrum status. Note that for the overlay strategy, $p_{a}=0$. Let $I \neq 0$ be the received interference at $\mathrm{Rx}_{s}$ imposed by $\mathrm{Tx}_{p}$. In cases where $I$ is large, it would be better to limit the access time during the busy period, i.e., decreasing $p_{a}$. However, in cases where $I$ is low, $p_{a}$ could be increased.

The optimal channel capacity of the secondary service when the secondary service accesses to spectrum by mixed strategy is the solution to the following optimization problem: 


\section{Problem $\mathcal{O}_{\text {opt }}$.}

$$
\begin{aligned}
C_{\mathrm{opt}}\left(p_{i}, I\right)= & \max _{P_{s, i} \geq 0, P_{s, b} \geq 0} p_{a} p_{b} \mathbf{E}_{\mathbf{g}_{s}} B \log \left(1+\frac{g_{s s} P_{s, b}}{N_{0} B+p_{a} I}\right) \\
& +p_{i} \mathbf{E}_{\mathbf{g}_{s}} B \log \left(1+\frac{g_{s s} P_{s, i}}{N_{0} B}\right), \\
\text { s.t. } \quad & p_{a} p_{b} \mathbf{E}_{\mathbf{g}_{s}} P_{s, b}+p_{i} \mathbf{E}_{\mathbf{g}_{s}} P_{s, i} \leq \bar{P}_{s}, \\
& \mathbf{E}_{\mathbf{g}_{s}} g_{s p} P_{s, b} \leq Q,
\end{aligned}
$$

where, $P_{s, i}$ and $P_{s, b}$ are the allocated power in the idle and busy periods, respectively. Note that in spectrum sharing, $P_{s, i}$ is only a function of $g_{s s}$, and $P_{s, b}$ is a function of $g_{s s}$ and $g_{s p}$. Finding a solution to $\mathcal{O}_{\mathrm{opt}}$ is rather difficult. In the following, we propose a straightforward approach to find an approximation of $C_{\mathrm{opt}}\left(p_{i}, I\right)$.

To solve $\mathcal{O}_{\text {opt }}$, we note that on one hand, during the active periods, the mixed strategy in $\mathcal{O}_{\text {opt }}$ acts similar to the underlay strategy; therefore, the achievable capacity is obtained through the following optimization problem:

$$
\begin{aligned}
C_{U}(I)= & p_{a} p_{b} \max _{P_{s, b} \geq 0} \mathbf{E}_{\mathbf{g}_{s}} B \log \left(1+\frac{g_{s s} P_{s, b}}{N_{0} B+p_{a} I}\right), \\
\text { s.t. } \quad & \mathbf{E}_{\mathbf{g}_{s}} P_{s, b} \leq \bar{P}_{s, b}, \\
& \mathbf{E}_{\mathbf{g}_{s}} g_{s p} P_{s, b} \leq Q,
\end{aligned}
$$

where $\bar{P}_{s, b}$ is the power constraints during the busy periods.

On the other hand, the mixed strategy in $\mathcal{O}_{\text {opt }}$ acts similar to the overlay strategy during the idle period; therefore, the achievable capacity is obtained through the following optimization problem:

$$
\begin{aligned}
C_{O}\left(p_{i}\right)= & p_{i} \max _{P_{s, i} \geq 0} \mathbf{E}_{\mathbf{g}_{s}} B \log \left(1+\frac{g_{s s} P_{s, i}}{N_{0} B}\right), \\
\text { s.t. } & \mathbf{E}_{\mathbf{g}_{s}} P_{s, i} \leq \bar{P}_{s, i},
\end{aligned}
$$

where $\bar{P}_{s, i}$, is the power constraints during the idle periods.

Therefore, $C_{\mathrm{opt}}\left(p_{i}, I\right)$ is approximated by

$$
C_{M}\left(p_{i}, I\right)=C_{O}\left(p_{i}\right)+C_{U}(I) .
$$

In this paper, we refer to $C_{\text {opt }}\left(p_{i}, I\right)$ as optimal channel capacity and $C_{M}\left(p_{i}, I\right)$ as maximum achievable capacity for brevity. It is shown in Section 5.2 that $C_{M}\left(p_{i}, I\right)$ is very close to the $C_{\mathrm{opt}}\left(p_{i}, I\right)$. Note that the transmission power of the secondary service during the idle and busy periods must satisfy the total power constraint as follows:

$$
p_{a} p_{b} \bar{P}_{s, b}+p_{i} \bar{P}_{s, i}=\bar{P}_{s} .
$$

The optimization problems (36) and (37) are related through (39). Therefore, to obtain the achievable capacity and the corresponding transmit power allocation, $\bar{P}_{s, b}$ and $\bar{P}_{s, i}$ should be evaluated.

\subsection{Evaluating Maximum Achievable Capacity}

Here, we propose a simple scheme to evaluate $\bar{P}_{s, b}$ and $\bar{P}_{s, i}$, thus obtaining a solution to $C_{M}\left(p_{i}, I\right)$. As it was shown in Section 3, for the underlay strategy, the transmission power of the secondary service could be allocated by adjusting the maximum transmission power constraint without loss of achievable capacity so that signaling between $\mathrm{Tx}_{s}$ and $\mathrm{Rx}_{p}$ is not required. Here, we extend the aforementioned approach for the proposed mixed strategy.

We start with the busy state and assume that $\bar{P}_{s, b}=\bar{P}_{s,}$ where $\bar{P}_{s}$ is the maximum transmit power of $\operatorname{Tx}_{s}$. Using $Q$, we then specify whether the system is interference-constrained or power-constrained. If it is a power-constrained system, the initial setting is correct and $\bar{P}_{s, i}$ is obtained from (39). If the spectrum sharing is interference-constrained, we obtain $\bar{P}_{s, b}$ so that the system is transformed to the powerconstrained region. Consequently, $\bar{P}_{s, i}$ is obtained using (39). The aforementioned proposed method to evaluate $\bar{P}_{s, b}$ and $\bar{P}_{s, i}$ is elaborated in the following.

Let $\bar{P}_{s, b}=\bar{P}_{s}$, then the critical interference threshold $\gamma_{Q}^{*}$ is obtained using (25) as

$$
\gamma_{Q}^{*}=\gamma_{P}^{b}+\left(\rho_{Q}^{b}-\frac{e^{-\rho_{P}^{b}}}{\rho_{P}^{b}}\right),
$$

where $\rho_{Q}^{b}$ and $\rho_{P}^{b}$ are, respectively, obtained similar to (15) and (22) as

$$
\rho_{Q}^{b}-\log \left(1+\rho_{Q}^{b}\right)=\gamma_{Q}, \quad \frac{e^{-\rho_{P}^{b}}}{\rho_{P}^{b}}-E_{1}\left(\rho_{P}^{b}\right)=\gamma_{P}^{b} .
$$

In (41), $\gamma_{Q} \triangleq Q /\left(N_{0} B+p_{a} I\right)$, and $\gamma_{P}^{b} \triangleq \bar{P}_{s, b} /\left(N_{0} B+p_{a} I\right)$. Having $Q$, and correspondingly $\gamma_{Q}$, if $\gamma_{Q} \geq \gamma_{Q}^{*}$, then the spectrum sharing is power-constrained, and using (39), we obtain

$$
\bar{P}_{s, b}=\bar{P}_{s}, \quad \bar{P}_{s, i}=\bar{P}_{s} \frac{1-p_{a} p_{b}}{p_{i}} .
$$

In case that $\gamma_{Q} \leq \gamma_{Q}^{*}$, the spectrum sharing is interferenceconstrained. Therefore, by adjusting $\bar{P}_{s, b}$, the system can be transformed into the power-constrained spectrum sharing. We showed in Section 3 that this transformation does not result in reducing the secondary service's achievable capacity. To obtain $\bar{P}_{s, b}$, we first use (40) and obtain $\gamma_{P}^{b}$ :

$$
\gamma_{P}^{b}=\gamma_{Q}^{*}-\left(\rho_{Q}^{b}-\frac{e^{-\rho_{P}^{b}}}{\rho_{P}^{b}}\right),
$$

and then through the definition $\gamma_{P}^{b} \triangleq \bar{P}_{s, b}\left(N_{0} B+p_{a} I\right)$, parameter $\bar{P}_{s, b}$ is obtained. Having $\bar{P}_{s, b}$, then $\bar{P}_{s, i}$ is also obtained using (39):

$$
\bar{P}_{s, i}=\frac{\bar{P}_{s}-p_{a} p_{b} \bar{P}_{s, b}}{p_{i}} .
$$

The above method to obtain $\bar{P}_{s, b}, \bar{P}_{s, i}$ can be summarized as follows:

$$
\begin{aligned}
& \bar{P}_{s, b}=\min \left(\bar{P}_{s}, \frac{\gamma_{Q}^{*}-\left(\rho_{Q}^{b}-\frac{e^{-\rho_{P}^{b}}}{\rho_{P}^{b}}\right)}{N_{0} B+p_{a} I}\right), \\
& \bar{P}_{s, i}=\frac{\bar{P}_{s}-p_{a} p_{b} \bar{P}_{s, b}}{p_{i}} .
\end{aligned}
$$

As seen in obtaining $\bar{P}_{s, b}$ and $\bar{P}_{s, i}$, parameter $g_{s p}$ is not required and only $g_{s s}$ appears. This is mainly due to the fact that in idle periods, the secondary service does not require $g_{s p}$ because there is no transmission from the primary service. Moreover, the proposed scheme during the busy periods 
keeps the spectrum sharing system to be power-constrained by adjusting the system parameters. Therefore, even in this case, $g_{s p}$ is not required for the power allocation.

Since in the proposed scheme, the spectrum sharing system is always power-constrained, the maximum achievable capacity of the secondary service is obtained from the following two optimization problems:

$$
\begin{aligned}
C_{U}(I)= & p_{a} p_{b} \max _{P_{s, b} \geq 0} \mathbf{E}_{\mathbf{g}_{s}} B \log \left(1+\frac{g_{s s} P_{s, b}}{N_{0} B+p_{a} I}\right), \\
& \mathbf{E}_{\mathbf{g}_{s}} P_{s, b} \leq \bar{P}_{s, b},
\end{aligned}
$$

and

$$
\begin{aligned}
C_{O}\left(p_{i}\right)= & p_{i} \max _{P_{s, i \geq 0}} \mathbf{E}_{\mathbf{g}_{s}} B \log \left(1+\frac{g_{s s} P_{s, i}}{N_{0} B}\right), \\
& \mathbf{E}_{\mathbf{g}_{s}} P_{s, i} \leq \bar{P}_{s, i} .
\end{aligned}
$$

The optimization problems ((47) and (49)) are samples of water-filling problem. Note that in (48) and (50), $\bar{P}_{s, b}$ and $\bar{P}_{s, i}$ are obtained from (45) and (46), respectively

The optimal power allocation in (47) is obtained as

$$
P_{s, b}^{*}=\left(\frac{p_{a} p_{b}}{\lambda_{b}^{*}}-\frac{N_{0} B+p_{a} I}{g_{s s}}\right)^{+},
$$

where $\lambda_{b}^{*}$ is the Lagrangian coefficient. By replacing $P_{s, b}^{*}$ in (48) and considering the equality, $\rho_{b}$ (or, equivalently, $\lambda_{b}^{*}$ ) is then obtained from the following:

$$
\frac{e^{-\rho_{b}}}{\rho_{b}}-E_{1}\left(\rho_{b}\right)=\frac{\bar{P}_{s, b}}{N_{0} B+p_{a} I}=\gamma_{P}^{b},
$$

where $\rho_{b} \triangleq \lambda_{b}^{*}\left(N_{0} B+p_{a} I\right) / p_{a} p_{b}$. Consequently, by replacing $P_{s, b}^{*}$ into (47), the achievable capacity is

$$
C_{U}(I)=p_{a} p_{b} B E_{1}\left(\rho_{b}\right)
$$

In the idle periods, the optimal transmission power is obtained from (49) as

$$
P_{s, i}^{*}=\left(\frac{p_{i}}{\lambda_{i}^{*}}-\frac{N_{0} B}{g_{s s}}\right)^{+},
$$

where $\lambda_{i}^{*}$ is the Lagrangian coefficient and is obtained from the following:

$$
\frac{e^{-\rho_{i}}}{\rho_{i}}-E_{1}\left(\rho_{i}\right)=\gamma_{P}^{i},
$$

where $\rho_{i} \triangleq \lambda_{i}^{*} N_{0} B / p_{i}$ and $\gamma_{P}^{i} \triangleq \bar{P}_{i} / N_{0} B$. The achievable capacity is then obtained by replacing $P_{s, i}^{*}$ into (49):

$$
C_{O}\left(p_{i}\right)=p_{i} B E_{1}\left(\rho_{i}\right) .
$$

Therefore, the total achievable capacity is obtained by replacing (53) and (56) into (38):

$$
C_{M}\left(p_{i}, I\right)=p_{i} B E_{1}\left(\rho_{i}\right)+p_{a} p_{b} B E_{1}\left(\rho_{b}\right),
$$

where $E_{1}(x) \triangleq \int_{1}^{\infty} t^{-1} e^{-x t} d t, \quad x \geq 0$.

\subsection{Numerical Result}

\subsubsection{Impact of Access Probability}

In Fig. 7, the impact of access probability, $p_{a}$, on the achievable capacity of the secondary service in the mixed strategy is

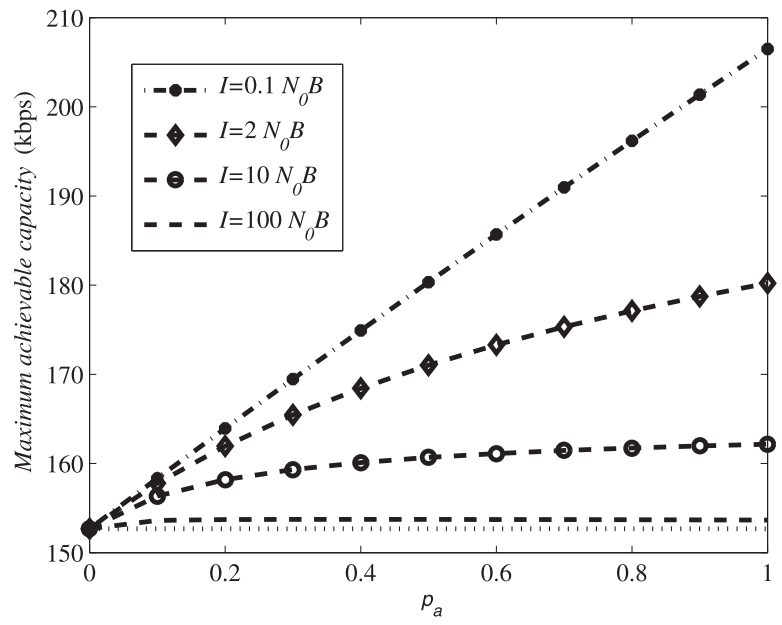

Fig. 7. The achievable capacity of the secondary service, where $\bar{P}_{s}=1 \mathrm{~W}$, $Q=2 N_{0} B$.

studied for different values of $I$, where $\bar{P}_{s}=1$ Watt and $Q=2 N_{0} B$. For the case that $p_{a}=0$, the secondary service accesses the spectrum only in the idle periods, i.e., overlay strategy. In the case that $p_{a}=1$, which we call modified underlay strategy, the secondary service always has access to the spectrum. It is called the modified underlay strategy, because here the spectrum sharing recognizes the idle and busy periods and during idle periods in contrast to the underlay strategy interference constraint is not considered.

As seen in Fig. 7, for a given value of $I$, the achievable capacity is a function of $p_{a}$. Also, it is seen that by increasing $I$, $C_{M}\left(p_{i}, I\right)$ is decreased. In the low-interference regime, i.e., $I<N_{0} B$, the achievable capacity is increased by increasing $p_{a}$. In the low-interference regime, both modified underlay (i.e., $p_{a}=1$ ) and mixed strategies (i.e., $0<p_{a}<1$ ) outperform the overlay strategy (i.e., $\left.p_{a}=0\right)$. As it is seen, in the lowinterference regime, $C_{M}\left(p_{i}, I\right)$ is almost a linear function of $p_{a}$.

For a moderate $I$, e.g., $I=2 N_{0} B$, by increasing $p_{a}$, $C_{M}\left(p_{i}, I\right)$ is still increased; however, the increment is made with a lower rate than that of in low-interference regime. In the high-interference regime, i.e., $I \gg N_{0} B$, Fig. 7 indicates that the rate of increasing $C_{M}\left(p_{i}, I\right)$ by $p_{a}$ is reduced, and for very high interference, the achievable capacity is not increased anymore for larger values of $p_{a}$, as it was expected. For instance, in the case that $I=10 N_{B}$, by increasing $p_{a}$ from 0 to $0.4, C_{M}\left(p_{i}, I\right)$ is increased and then remains constant for higher values of $p_{a}$.

As seen in Fig. 7, in the high-interference regime, only accessing with a low value of $p_{a}$ is beneficial. In other words, in such cases, the overlay strategy performs approximately similar to the mixed strategy. However, in the low-interference regime, the access to the busy periods even with a high $p_{a}$ increases the achievable capacity. In other words, for very low interference, larger $p_{a}$ results in higher capacity, i.e., the underlay behavior of the mixed strategy is dominant and for very high interference, smaller $p_{a}$ results in higher capacity, i.e., the overlay behavior of the mixed strategy is dominant.

\subsubsection{Comparing Underlay and Modified Underlay Strategies}

As shown in Fig. 8, the achievable capacity of the secondary service with the modified underlay strategy is significantly 


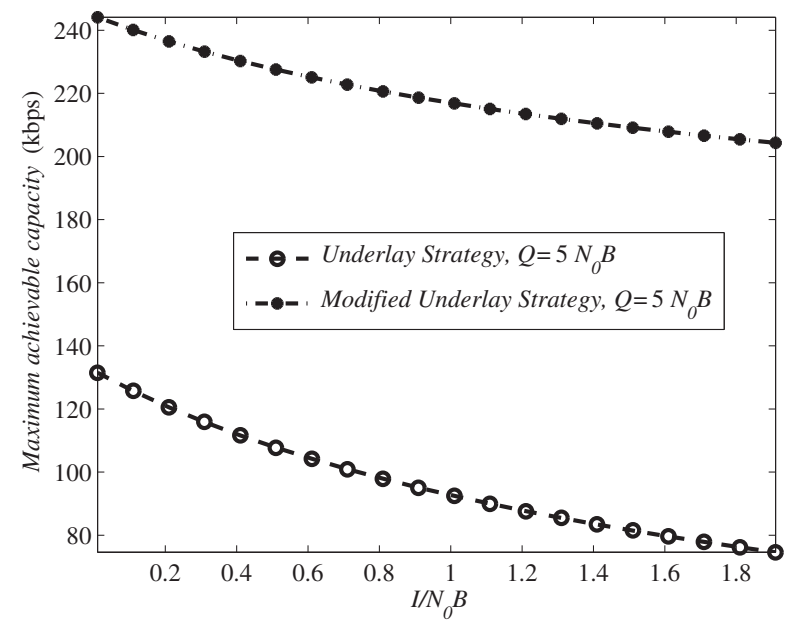

Fig. 8. The achievable capacity of the secondary service for the underlay and modified underlay strategies for $\bar{P}_{s}=1 \mathrm{~W}$ and $p_{i}=0.3$.

larger than the achievable capacity in the underlay strategy. Therefore, the modified underlay strategy always outperforms the underlay strategy.

\subsubsection{Comparing $C_{M}$ and $C_{\text {opt }}$}

The mixed strategy based on the proposed power allocation scheme always outperforms the underlay strategy especially in the form of modified underly strategy. Furthermore, its performance is often higher than that of the underlay strategy. Therefore, the mixed strategy based on the proposed power allocation scheme achieves higher capacity than that of other access strategies.

Here, our objective is to investigate whether the achieved capacity $C_{M}\left(p_{i}, I\right)$, can reach the maximum achievable capacity of the channel, $C_{\mathrm{opt}}\left(p_{i}, I\right)$, by using the proposed power allocation scheme. We refer to $C_{\mathrm{opt}}\left(p_{i}, I\right)$ as the optimal channel capacity. As indicated in Fig. 9, $C_{M}\left(p_{i}, I\right) \neq$ $C_{\text {opt }}\left(p_{i}, I\right)$ for large enough $Q$, and for small or moderate $Q$, $C_{M}\left(p_{i}, I\right) \approx C_{\text {opt }}\left(p_{i}, I\right)$. In practice, if $Q$ falls in the category of small and moderate $Q$; then, in the proposed mixed strategy, the secondary service achieves capacity very close to the optimal channel capacity.

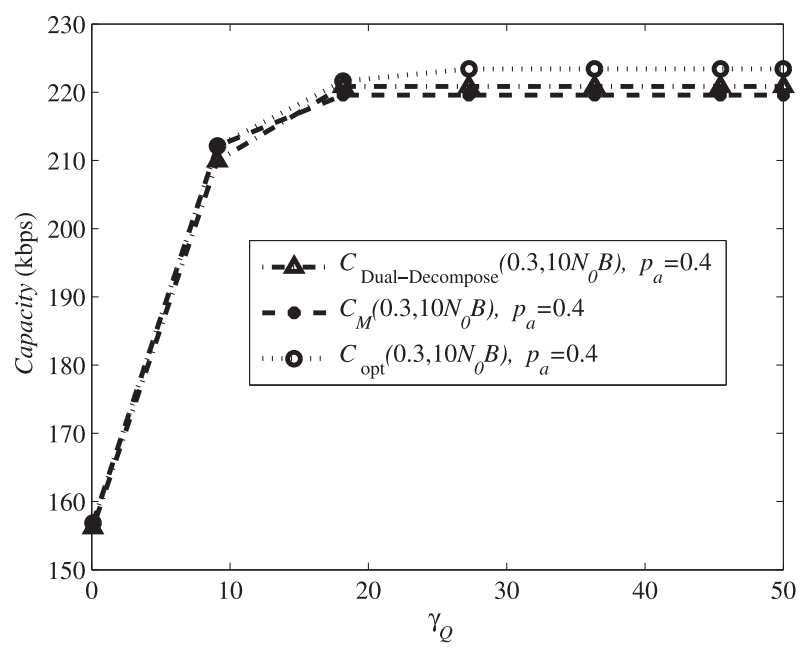

Fig. 9. The optimal channel capacity and the achievable capacity of the mixed strategy where $\bar{P}_{s}=1 \mathrm{~W}$.

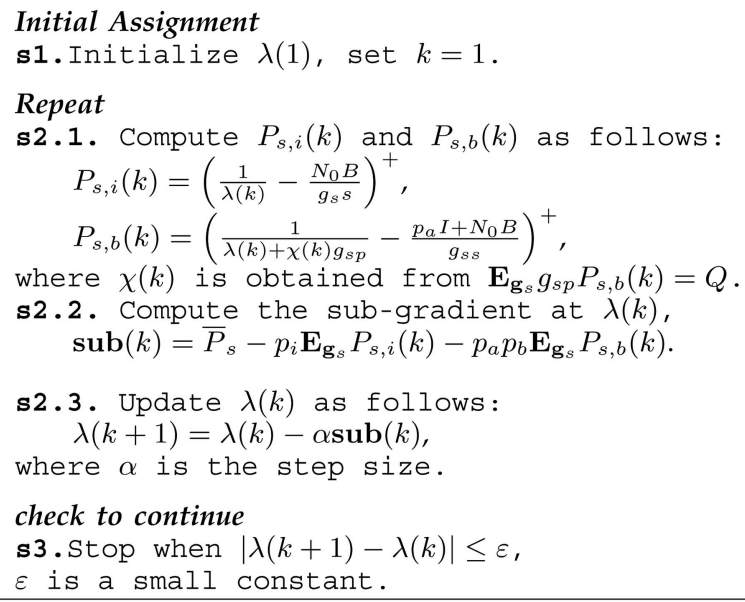

Fig. 10. The heuristical algorithm based on dual decomposition method [24].

Here, we also compare the performance of the proposed power allocation scheme with the one that was presented in [24]. For easy reference, in Fig. 10, we represent a revised version of the algorithm in [24] based on our system model and parameters. This heuristic algorithm is based on dualdecomposition methods. As seen in Fig. 9, our proposed power allocation scheme has approximately the same performance as the heuristic algorithm proposed in [24]. It must be noted that our proposed scheme for allocating power is significantly less complex than that presented in [24]. Furthermore, we obtain a closed form for the achievable capacity.

\section{Conclusions}

In this paper, we analyzed the corresponding secondary service achievable capacity for overlay and underlay access strategies. We then obtained a critical system parameter for underlay spectrum sharing to eliminate the interference threshold constraint which significantly reduced the system complexity by making the power allocation of the secondary service independent from the channel-side information between the secondary transmitter and the primary receiver; thus removing the need for signaling between primary and secondary systems. For underlay spectrum sharing, we analyzed the achievable capacity for accurate and inaccurate spectrum sensing.

We then noted that the overlay strategy ignored the interference tolerability of the primary network. The underlay strategy also ignored the opportunity lying in monitoring the activity of the primary network, in which in the idle periods, if detected properly, the secondary service might be able to transmit without interference threshold constraints. Hence, in both underlay and overlay strategies, system-inherent opportunities, which could be exploited to improve the achievable capacity of the secondary service, were missed.

To exploit the aforementioned features of the primary service, we then proposed a novel mixed-access strategy in which during idle periods, in contrast to the underlay 
strategy, the secondary service transmits without considering the interference threshold constraint. In contrast to the overlay strategy, during the busy periods, in the proposed mixed strategy the secondary service made transmission with a probability $p_{a}$ subject to satisfying the interference threshold constraint. Parameter $p_{a}$ is a secondary service parameter which can be adjusted based on the spectrum status. We then obtained the achievable capacity of the proposed mixed strategy.

Moreover, we proposed a simple power allocation scheme for the mixed strategy wherein its corresponding achieved capacity was very close to the optimal channel capacity of the secondary service. Through simulations, we further showed that based on the imposed interference at the secondary service receiver from the primary service transmitter, $I$, the secondary service can adjust $p_{a}$ to choose its access strategy and maximize the achieved capacity. The proposed approach to the spectrum sharing based on $I$ significantly reduced the system complexity compared to the system in which for spectrum sharing the imposed interference at the primary receiver was required.

Analyzing access strategies for spectrum sharing systems with multiple primary and secondary users can be considered as a future work.

\section{ACKNOWLEDGMENTS}

The work of K. Navaie and M.G. Khoshkholgh is supported in part by $\mathrm{MCI}$, under contract TMU-87-12-87. The corresponding author is Keivan Navaie.

\section{REFERENCES}

[1] J.M. Peha, "Approaches to Spectrum Sharing," IEEE Comm. Magazine, vol. 43, no. 2, pp. 10-12, Feb. 2005.

[2] S. Haykin, "Cognitive Radio: Brain-Empowered Wireless Communications," IEEE J. Selected Areas in Comm., vol. 23, no. 2, pp. 201-220, Feb. 2005.

[3] Q. Zhao and B. Sadler, "A Survey of Dynamic Spectrum Access: Signal Processing, Networking, and Regulatory Policy," IEEE Signal Processing Magazine, vol. 24, no. 3, pp. 79-89, May 2007.

[4] X. Liu and S.N. Shankar, "Sensing-Based Opportunistic Channel Access," ACM J. Mobile Networks and Applications, vol. 11, no. 4, pp. 577-591, Aug. 2006.

[5] R. Tandra, S.M. Mishra, and A. Sahai, "What Is a Spectrum Hole and What Does It Take to Recognize One?" Proc. IEEE, vol. 97, no. 5, pp. 824-848, May 2009.

[6] "Spectrum Policy Taskforce Report," technical report, Fed. Comm. Commission, http://hraunfoss.fcc.gov/edocs_public/ attachmatch/DOC-228542A1.pdf, Nov. 2002.

[7] M. Gastpar, "On Capacity Under Receive and Spatial SpectrumSharing Constraints," IEEE Trans. Information Theory, vol. 53, no. 2, pp. 471-487, Feb. 2007.

[8] S.A. Jafar and S. Srinivasa, "Capacity Limits of Cognitive Radio with Distributed and Dynamic Spectral Activity," IEEE J. Selected Areas in Comm., vol. 25, no. 3, pp. 529-537, Apr. 2007.

[9] A. Ghasemi and E.S. Sousa, "Fundamental Limits of SpectrumSharing in Fading Environments," IEEE Trans. Wireless Comm., vol. 6, no. 2, pp. 649-658, Feb. 2007.

[10] G. Bansal, M.J. Hossain, and V.K. Bhargava, "Optimal and Suboptimal Power Allocation Schemes for OFDM-Based Cognitive Radio Systems," IEEE Trans. Wireless Comm., vol. 7, no. 11, pp. 47104718, Nov. 2008.

[11] L. Musavian and S. Aissa, "Capacity and Power Allocation for Spectrum-Sharing Communications in Fading Channels," IEEE Trans. Wireless Comm., vol. 8, no. 1, pp. 148-156, Jan. 2009.

[12] S. Sridharan and S. Vishwanath, "On the Capacity of a Class of MIMO Cognitive Radios," IEEE J. Selected Topics in Signal Processing, vol. 2, no. 1, pp. 103-117, Feb. 2008.
[13] D. Cabric, I.D. O'Donnell, M.S.W. Chen, and R.W. Brodersen, "Spectrum Sharing Radios," IEEE Circuits and Systems Magazine, vol. 6, no. 2, pp. 30-45, July 2006.

[14] R. Zhang, “On Peak versus Average Interference Power Constraints for Protecting Primary Users in Cognitive Radio Networks," IEEE Trans. Wireless Comm., vol. 8, no. 4, pp. 2112-2120, Apr. 2009.

[15] W.Y. Lee and I.F. Akyildiz, "Optimal Spectrum Sensing Framework for Cognitive Radio Networks," IEEE Trans. Wireless Comm., vol. 7, no. 10, pp. 3845-3857, Oct. 2008.

[16] Z. Quan, S. Cui, and A.H. Sayed, "Optimal Linear Cooperation for Spectrum Sensing in Cognitive Radio Networks," IEEE J. Selected Topics in Signal Processing, vol. 2, no. 1, pp. 28-40, Feb. 2008.

[17] S. Tang and B. Mark, "Modeling and Analysis of Opportunistic Spectrum Sharing with Unreliable Spectrum Sensing," IEEE Trans. Wireless Comm., vol. 8, no. 4, pp. 1934-1943, Apr. 2009.

[18] Y.C. Liang, Y. Zeng, E.C.Y. Peh, and A.T. Hoang, "SensingThroughput Tradeoff for Cognitive Radio Networks," IEEE Trans. Wireless Comm., vol. 7, no. 4, pp. 1326-1337, Apr. 2008.

[19] Y. Chen, Q. Zhao, and A. Swami, "Joint Design and Separation Principle for Opportunistic Spectrum Access in the Presence of Sensing Errors," IEEE Trans. Information Theory, vol. 54, no. 5, pp. 2053-2071, May 2008.

[20] S. Huang, X. Liu, and Z. Ding, "Optimal Transmission Strategies for Dynamic Spectrum Access in Cognitive Radio Networks," IEEE Trans. Mobile Computing, vol. 8, no. 12, pp. 1636-1648, Dec. 2009.

[21] D. Zhang, Z. Tian, and G. Wei, "Spatial Capacity of Narrowband vs. Ultra-Wideband Cognitive Radio Systems," IEEE Trans. Wireless Comm., vol. 7, no. 11, pp. 4670-4680, Nov. 2008.

[22] M.G. Khoshkholgh, K. Navaie, and H. Yanikomeroglu, "On the Impact of the Primary Network Activity on the Achievable Capacity of Spectrum Sharing over Fading Channels," IEEE Trans. Wireless Comm., vol. 8, no. 4, pp. 2100-2111, Apr. 2009.

[23] R. Menon, R.M. Buehrer, and J.H. Reed, "On the Impact of Dynamic Spectrum Sharing Techniques on Legacy Radio Systems," IEEE Trans. Wireless Comm., vol. 7, no. 11, pp. 4198-4207, Nov. 2008.

[24] X. Kang, Y.C. Liang, H.K. Garg, and L. Zhang, "Sensing-Based Spectrum Sharing in Cognitive Radio Networks," IEEE Trans. Vehicular Technology, vol. 58, no. 8, pp. 4649-4654, Oct. 2009.

[25] Y. Chen, G. Yu, Z. Zhang, H.H. Chen, and P. Qiu, “On Cognitive Radio Networks with Opportunistic Power Control Strategies in Fading Channels," IEEE Trans. Wireless Comm., vol. 7, no. 7, pp. 2752-2761, July 2008.

[26] X. Kang, Y.C. Liang, H.K. Garg, and R. Zhang, “Optimal Power Allocation for Fading Channels in Cognitive Radio Networks: Ergodic Capacity and Outage Capacity," IEEE Trans. Wireless Comm., vol. 8, no. 2, pp. 940-950, Feb. 2009.

[27] L. Gao and S. Cui, "Power and Rate Control for DelayConstrained Cognitive Radios via Dynamic Programming," IEEE Trans. Vehicular Technology, vol. 58, no. 9, pp. 4819-4827, Nov. 2009.

[28] T.M. Cover and J.A. Thomas, Elements of Information Theory, second ed. John Wiley and Sons, 2006.

[29] M.S. Alouini and A.J. Goldsmith, "Capacity of Rayleigh Fading Channels under Different Adaptive Transmission and DiversityCombining Techniques," IEEE Trans. Vehicular Technology, vol. 48, no. 4, pp. 1165-1181, July 1999.

[30] A. Ghasemi and E.S. Sousa, "Spectrum Sensing in Cognitive Radio Networks: Requirements Challenges and Design Trade-Offs," IEEE Comm. Magazine, vol. 46, no. 4, pp. 32-39, Apr. 2008.

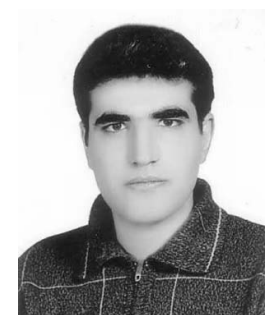

Mohammad G. Khoshkholgh received the BSc degree in electrical engineering from Isfahan University, Iran, in 2006, and the MSc degree in electrical engineering from Tarbiat Modares University, Tehran, Iran, in 2008 . He is currently working toward the $\mathrm{PhD}$ degree at Tarbiat Modares University. His research interests are mainly in wireless communications, radio resource allocations, and spectrum sharing. $\mathrm{He}$ is a student member of the IEEE. 


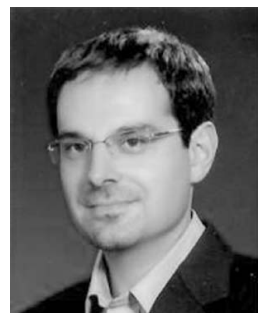

Keivan Navaie received the BSc degree from the Sharif University of Technology, Tehran, Iran, the MSc degree from the University of Tehran, Iran, and the PhD degree from Tarbiat Modares University, Tehran, Iran, in 1995, 1997, and 2004, respectively, all in electrical engineering. From March to November 2004, he was a postdoctoral research fellow with the School of Mathematics and Statistics, Carleton University, Ottawa, Canada. From December 2004 to September 2006, he was with the Broadband Communication and Wireless Systems (BCWS) Centre, Carleton University, Ottawa, Canada, where he was the project manager of BCWS participation in European Union Sixthth Framework Integrated Project, the Wireless World Initiative New Radio (WINNER) on Beyond 3G Wireless Systems. Since September 2006, he has been an assistant professor in the Department of Electrical and Computer Engineering, Tarbiat Modares University, Tehran, Iran. He is also the founder and director of the Wireless Innovation Laboratory (WIL), Tarbiat Modares University, Tehran, Iran. He has been a member of the Technical Committee of WASA 2006, the 16th Iranian Conference on Electrical Engineering (ICEE 2007), the IEEE WCNC 2008, the IEEE GlobeCom 2008, the IEEE WiMob 2008, the IEEE WCNC 2009, the CNSR 2009, the IEEE WCNC 2010, and the CNSR 2010. His research interests are mainly in the resource management issues of cellular and multihop wireless networks, cooperative communications, spectrum sharing, and performance evaluation. He is a senior member of the IEEE.

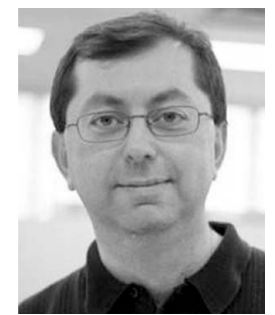

Halim Yanikomeroglu received the BSc degree in electrical and electronics engineering from the Middle East Technical University, Ankara, Turkey, in 1990, and the MASc degree in electrical engineering (now electrical and computer engineering) and the $\mathrm{PhD}$ degree in electrical and computer engineering from the University of Toronto, Canada, in 1992 and 1998, respectively. He was with the R\&D Group of Marconi Kominikasyon A.S., Ankara, Turkey, from 1993 to 1994. Since 1998, he has been with the Department of Systems and Computer Engineering, Carleton University, Ottawa, where he is currently a full professor. His research interests cover many aspects of the physical, medium access, and networking layers of wireless communications. His research is currently funded by Samsung (SAIT, Korea), Huawei (China), the Communications Research Center Canada (CRC), and the NSERC. He is a recipient of the Carleton University Research Achievement Award 2009. He has been involved in the steering committees and technical program committees of numerous international conferences. He has also given 17 tutorials in such conferences. He is a member of the steering committee of the IEEE Wireless Communications and Networking Conference (WCNC), and has been involved in the organization of this conference over the years, including serving as the technical program cochair of WCNC 2004 and the technical program chair of WCNC 2008. He was the general cochair of the IEEE Vehicular Technology Conference held in Ottawa in September 2010 (VTC2010-Fall). He was an editor for the IEEE Transactions on Wireless Communications (2002-2005) and the IEEE Communications Surveys \& Tutorials (2002-2003), and a guest editor for the Wiley Journal on Wireless Communications \& Mobile Computing. He was an officer of the IEEE's Technical Committee on Personal Communications (Chair: 2005-2006, Vice-Chair: 2003-2004, Secretary: 2001-2002), and he was also a member of the IEEE Communications Society's Technical Activities Council (2005-2006). He is also an adjunct professor at King Saud University, Riyadh, Saudi Arabia. $\mathrm{He}$ is a member of the Carleton University Senate and a registered professional engineer in the province of Ontario, Canada. He is a member of the IEEE.

$\triangleright$ For more information on this or any other computing topic, please visit our Digital Library at www.computer.org/publications/dlib. 